\title{
How does sediment exposure affect corals? A systematic review protocol
}

\author{
Lillian J. Tuttle ${ }^{1 *}\left(\mathbb{D}\right.$, Craig Johnson ${ }^{2}$, Steve Kolinski ${ }^{3}$, Dwayne Minton ${ }^{4}$ and Megan J. Donahue ${ }^{1}$
}

\begin{abstract}
Background: Local management action to address coral-reef stressors can improve reef health and mitigate the effects of global climate change. Coastal development and runoff lead to sedimentation, which directly impacts coral recruitment, growth, mortality, and the ecosystem services that coral reefs provide. Decision making for reef resilience in the face of global and local stressors requires information on thresholds for management action. In response to needs identified by reef managers, we plan to conduct a systematic review and meta-analysis that will explore the effects of both deposited and suspended sediment on corals to identify single and interacting stressor thresholds. We will identify levels of sediment exposure (i.e., concentration, duration, and frequency) that cause adverse physical, physiological, behavioral, developmental, and ecological responses in coral and describe geographic and taxonomic patterns in these responses. Our ultimate goal is to provide managers with sediment exposure thresholds that can be expected to cause these responses.

Methods: Our systematic review will synthesize available evidence on the effects of suspended and deposited sediment on corals. The research questions were formulated with an advisory team to support management decisions concerning local reef stressors in waters under U.S. federal jurisdiction. While the advisory team is most concerned with reefs adjacent to U.S. Pacific Islands, our review will include studies that examine reef-building coral species around the world. We will search online databases and grey literature to obtain a list of potential studies, assess their relevance, and critically appraise them for validity and risk of bias. Provided enough data can be extracted from relevant experimental studies, we will conduct meta-analyses that examine changes in coral health and survival in response to suspended and/or deposited sediment, with the goal to define sediment thresholds for reef managers. If enough data are available from within the U.S. Pacific Islands, we will construct region-, site-, and/or species-specific thresholds to improve local management.
\end{abstract}

Keywords: Sedimentation, Turbidity, Dredging, Marine management, Bleaching, Mortality, Sublethal physiology, Regulatory processes

\section{Background}

Half of the world's coral reefs have been lost in recent decades [1-4], while rising sea surface temperatures and local stressors threaten one-third of those remaining [5]. This decline threatens the ecosystem services that reefs provide [6], including a USD $\$ 36$ billion annual tourism industry [7]. In the U.S. and areas under its

\footnotetext{
*Correspondence: tuttlel@hawaii.edu

${ }^{1}$ Hawai'i Institute of Marine Biology, Kāne'ohe, Hawai'i 96744, USA

Full list of author information is available at the end of the article
}

jurisdiction, corals and coral reefs are protected as federal trust resources, as special aquatic sites, for their value as habitat for fish, and because some corals are listed as threatened or endangered species [8-10]. The regulatory programs that apply to corals and coral reefs manage a wide variety of local stressors that include physical destruction and alteration; sediment, nutrients and chemical pollutants; and point sources of thermal pollution [8-10]. Other regulatory programs are designed to conserve species that use coral reefs as habitat and indirectly benefit reefs [11].

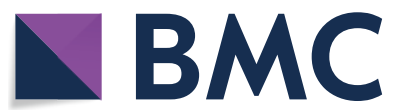

(c) The Author(s) 2020. This article is licensed under a Creative Commons Attribution 4.0 International License, which permits use, sharing, adaptation, distribution and reproduction in any medium or format, as long as you give appropriate credit to the original author(s) and the source, provide a link to the Creative Commons licence, and indicate if changes were made. The images or other third party material in this article are included in the article's Creative Commons licence, unless indicated otherwise in a credit line to the material. If material is not included in the article's Creative Commons licence and your intended use is not permitted by statutory regulation or exceeds the permitted use, you will need to obtain permission directly from the copyright holder. To view a copy of this licence, visit http://creativeco mmons.org/licenses/by/4.0/. The Creative Commons Public Domain Dedication waiver (http://creativecommons.org/publicdomain/ zero/1.0/) applies to the data made available in this article, unless otherwise stated in a credit line to the data. 
Management of coastal activities can minimize the degradation of water quality and bottom habitat, and thus mitigate reef decline in the face of climate change $[12,13]$. However, reefs face a litany of local stressors that may act synergistically and thus complicate regulatory programs [14]. Among the most damaging pollutants on coral reefs is sediment, which can remain suspended in the water or be deposited on the coral surface (i.e., turbidity and sedimentation, respectively) and can contain toxicants, pathogens, and nutrients, all of which impact coral growth, recruitment, and survival [15-18]. There is enormous variation in the levels of exposure to turbidity and sedimentation that corals can tolerate, which may result from taxonomic differences, geographic location, sediment type, and exposure concentration, duration, and frequency. Exploring potential sources of this variation will help to quantify synergistic effects and identify critical threshold values for sediment and other anthropogenic stressors on reef-building corals, thus enhancing efforts to conserve and restore coral reefs.

The Pacific Islands Regional Office (PIRO) of the U.S's National Oceanic and Atmospheric Administration (NOAA) is in the process of developing a tool to help regulators and the regulated community assess the effects of human activities on coral, coral reefs, and associated benthic communities in the Pacific and to develop appropriate measures to mitigate for unavoidable impacts. This 'Coral Tool' becomes more valuable if critical threshold values for suspended and deposited sediment on coral reefs can be identified; the tool currently relies on the results of previously published literature syntheses. The most widely cited reviews of the effects of coastal development [18] and terrestrial runoff [16] on coral reefs are now more than a decade old. Substantial new experimental data are now available to inform best management practices. More recent syntheses of the effects of sediment on corals $[15,17,19,20]$ provide qualitative accounts only, thus providing a starting point for the quantitative synthesis that allows regulatory assessments to rigorously identify thresholds and quantify adverse effects.

In response to needs identified by NOAA PIRO, we plan to conduct a systematic review and meta-analysis that will identify thresholds of coral response to both deposited and suspended sediment. These thresholds will be applied to NOAA's 'Coral Tool', bringing to bear the most current and comprehensive information for decision-making. Specific research questions and a protocol (see below) were developed by the team at NOAA that is building the Coral Tool (authors Johnson, Kolinski, and Minton, hereafter referred to as the 'Coral Tool advisory team') in conjunction with a research team from the
University of Hawai'i (authors Tuttle and Donahue), who will conduct the systematic review and meta-analysis.

\section{Objective of the review}

The primary objective of the present study is to perform a systematic review of peer-reviewed, public, and/or grey literature to develop thresholds for suspended and deposited sediment stressors that affect nearshore coral-reef ecosystems. We will follow established methodologies [21-23] for systematic review in environmental management to (a) identify, collect, and evaluate sources of empirical data on the effects of sediment on corals, both around the world and in the Pacific Islands Region; (b) extract relevant data from these sources using a common set of procedures; (c) organize and store these data for further analysis; and (d) use statistical analyses and metaanalytic procedures to identify single-stressor thresholds and multiple-stressor synergisms on coral reefs.

To disentangle the effects of synergistic stressors, we will focus on experimental studies that quantify the causal relationship between sediment and coral response. Monitoring and other observational studies will be used to contextualize experimental findings. We will address the following question and subquestions:

1. How does sediment exposure affect corals?

(a) What physical, physiological, behavioral, developmental, and ecological responses of corals are associated with sediment exposure (i.e., concentration, duration, or frequency)?

(b) What is the relationship between sediment exposure and the frequency and magnitude of coral responses (e.g., mortality, tissue necrosis, growth rate, photosynthetic yield, etc.)?

(c) How do coral responses to sediment exposure differ between deposited and suspended sediment?

(d) How do coral responses to sediment exposure differ by geography, sediment type, and coral taxonomy, morphology, and developmental stage?

\section{Methods}

Our systematic review and meta-analysis will be conducted according to the Guidelines and Standards for Evidence Synthesis in Environmental Management, version $5.0[21,22]$ and reported according to the procedures of ROSES (RepOrting standards for Systematic Evidence Syntheses) [23, Additional file 1].

\section{Searching for articles}

Our systematic review will start with the definitive reviews on the subject, which include Rogers [18], Fabricius [16], Erftemeijer et al. [15], Risk [19], and Jones 
et al. [17, 20]. We will develop a list of potential sources of data, hereafter called 'benchmark studies', from this set of reviews [Additional file 2].

To supplement this list, we will conduct electronic literature searches using the following databases or search engines (DSE) using the University of Hawai'i Mānoa Library: (1) ISI Web of Science (All Databases, see Table 1), (2) JSTOR, (3) Aquatic Sciences and Fisheries Abstracts, (4) Dissertations and Theses Global, (5) James Cook University Library One Search, (6) ReefBase's Proceedings of the International Coral Reef Symposium, (7) Science.gov, (8) Great Barrier Reef Marine Park Authority (GBRMPA) Elibrary, and (9) Western Australia Marine Science Institute's Dredging Science Node (WAMSI DSN) repository. These DSE are categorized and described in Table 1, along with search specifications (e.g., full text vs. abstract only, date ranges) for each. DSE 1-3 target peerreviewed literature produced by commercial publishers, while DSE 3-9 target 'grey' literature, including theses/ dissertations, conference proceedings, and reports for governmental/non-governmental entities.

The following genera will be specifically important because they contain species that are identified by the ESA as either threatened or endangered: Acropora, Anacropora, Cantharellus, Dendrogyra, Euphyllia, Isopora, Montastraea, Montipora, Mycetophyllia, Orbicella, Pavona, Porites, Seriatopora, Siderastrea, and Tubastraea. These additional genera will be important because of their importance in the Pacific Islands Region: Alveopora, Astreopora, Favia, Favites, Goniastrea, Goniopora, Leptastrea, Leptoria, Lobophyllia, Millepora, Platygyra, Pocillopora, and Turbinaria. The following search, in English, uses Boolean operators and wildcards to improve the quality (i.e., true positive results) of search results, and has been tested for its comprehensiveness [Additional file 2]: ((coral AND sediment*) OR (coral AND suspend*) OR (coral AND turbidity) OR (coral AND mud) OR (coral AND terrigenous) OR (coral AND silt*) OR (coral AND plume) OR (coral AND dredg*) OR (coral AND land-based) OR (sediment* AND Acropora) OR (sediment* AND Anacropora) OR (sediment* AND Cantharellus) OR (sediment* AND Dendrogyra) OR (sediment* AND Euphyllia) OR (sediment* AND Isopora) OR (sediment* AND Montastraea) OR (sediment* AND Montipora) OR (sediment* AND Mycetophyllia) OR (sediment* AND Orbicella) OR (sediment* AND Pavona) OR (sediment* AND Porites) OR (sediment" AND Seriatopora) OR (sediment* AND Siderastrea) OR (sediment* AND Tubastraea) OR (sediment* AND Alveopora) OR (sediment* AND Astreopora) OR (sediment* AND Favia) OR (sediment* AND Favites) OR (sediment* AND Goniastrea) OR (sediment* AND Goniopora) OR (sediment* AND Leptastrea) OR (sediment* AND Leptoria) OR (sediment* AND Lobophyllia) OR (sediment* AND Millepora) OR (sediment* AND Platygyra)

Table 1 Search specifications for each database or search engine (DSE)

\begin{tabular}{|c|c|c|c|c|}
\hline DSE Category & DSE Name (Abbrev.) & DSE Scope & Search specification(s) & Search dates \\
\hline \multirow[t]{4}{*}{ Bibliographic databases: } & $\begin{array}{l}\text { 1. Web of Science (WoS), All } \\
\text { Databases }\end{array}$ & General science & $\begin{array}{l}\text { Topic (titles, authors, abstracts, } \\
\text { keywords); 'All Databases' } \\
\text { include: (a) WoS Core Collec- } \\
\text { tion (SCI-EXPANDED, ESCI), } \\
\text { (b) Biological Abstracts, (c) } \\
\text { SciELO Citation Index, and (d) } \\
\text { Zoological Record }\end{array}$ & All years (1950-present) \\
\hline & 2. JSTOR & General academic & Abstract, All content & Any time \\
\hline & $\begin{array}{l}\text { 3. Aquatic Sciences and Fisheries } \\
\text { Abstracts (ASFA) }\end{array}$ & Aquatic and marine science & Abstract & Any time \\
\hline & $\begin{array}{l}\text { 4. Dissertations and Theses } \\
\text { Global (PQDT) }\end{array}$ & Global dissertations and theses & Abstract & Any time \\
\hline \multirow[t]{5}{*}{ Organizational databases: } & $\begin{array}{l}\text { 5. James Cook University One } \\
\text { Search (JCU) }\end{array}$ & $\begin{array}{l}\text { Australian university disserta- } \\
\text { tions and theses }\end{array}$ & Abstract, Dissertation/Thesis & Any time \\
\hline & 6. ReefBase & $\begin{array}{l}\text { Proceedings of the Interna- } \\
\text { tional Coral Reef Symposium }\end{array}$ & $\begin{array}{l}\text { Title; also Keywords for taxon- } \\
\text { specific search terms }\end{array}$ & Any time \\
\hline & 7. Science.gov & $\begin{array}{l}\text { United States federal govern- } \\
\text { ment science }\end{array}$ & $\begin{array}{l}\text { Full record (no 'Abstract' } \\
\text { option) }\end{array}$ & Any time \\
\hline & $\begin{array}{l}\text { 8. Great Barrier Reef Marine Park } \\
\text { Authority (GBRMPA) Elibrary }\end{array}$ & $\begin{array}{l}\text { Australian federal government } \\
\text { science }\end{array}$ & All of ELibrary, Type $=$ Report & Any time \\
\hline & $\begin{array}{l}\text { 9. Western Australia Marine } \\
\text { Science Institute's Dredging } \\
\text { Science Node (WAMSI DSN) }\end{array}$ & $\begin{array}{l}\text { Australian non-governmental } \\
\text { reports }\end{array}$ & $\begin{array}{l}\text { All reports and research articles } \\
\text { listed at [38] }\end{array}$ & Any time \\
\hline
\end{tabular}


OR (sediment* AND Pocillopora) OR (sediment* AND Turbinaria)).

Search results will be saved as BibTeX (.bib) or RIS (.ris) files and imported into open-source reference managers (e.g., Mendeley) with tools to identify and remove duplicates. We will test the thoroughness of our DSE searches by comparing the DSE search results with those of two other lists of potential sources of data. First, we will query Google Scholar with the same search string (using the Publish or Perish software tool [24] to export.ris files) then evaluate the top 1000 search results to include only 'relevant' articles (see Screening Process, below) and those un-duplicated in the DSE search. Similarly, we will screen the list of benchmark studies (described above) to include only relevant, un-duplicated articles [Additional file 2]. We will examine all relevant, un-duplicated articles within the Google Scholar search and the list of benchmark studies to understand why they were not also found in the DSE search. Possible reasons may include omission of certain journals or conference proceedings from database archives, narrow data ranges, and regional/language biases.

Based on any other systematic patterns of bias that we discern, we will reassess our DSE to be more inclusive. For instance, to avoid regional/language biases, we have included the SciELO Citation Index in the Web of Science search [DSE 1] that targets Latin American research in many Caribbean countries where we expect relevant work to be based.

\section{Article screening and study eligibility criteria Screening process}

For the purposes of this systematic review, an "article" is defined as any written document including scientific papers, abstracts, reports, book chapters, theses/dissertations, and other publications. Unique articles will be imported into abstrackr [25], a free web application in which the results of a literature search for a systematic review can be uploaded, organized, and screened. All reviewers will independently screen a pilot round of 100 articles (titles and abstracts evaluated together), classifying each as 'relevant,' 'irrelevant,' or 'maybe relevant' to the research question. The reviewers will discuss any discrepancies in their decisions and further clarify, revise, and agree upon the classification criteria until a consensus is reached for each conflict. Subsequently, all articles will be independently screened by at least two reviewers, and any conflicts between the two reviewers will be resolved by a third member of the review team. If a potential article was authored or co-authored by a reviewer, then two other reviewers will determine the potential relevance of the article. This will be done during the full-text screening as well (see below).
As the reviewers continue to make decisions about the articles' relevance, abstrackr's machine learning protocol predicts relevant articles and presents them to the reviewer(s) in order from 'most likely' to 'least likely' to be relevant. This can increase workload savings while maintaining relatively high sensitivity and specificity and relatively low false-negative rates, thus making it a useful addition to the screening process [26, 27]. Regardless of abstrackr's prediction, the reviewer(s) will screen all titles and abstracts. We will consider English abstracts for non-English full texts during the article screening process. When a non-English article is deemed potentially relevant, we will search for translations of full texts. If English translations are not available, the article will not be screened.

The full texts for all 'relevant' and 'maybe relevant' articles will be collected and reviewed according to the 'Eligibility Criteria' described below. Full-text screening will be conducted by one reviewer. A second reviewer will screen $10 \%$ of the full texts and compare their decisions with that of the initial reviewer. If the two reviewers have conflicting decisions, they will discuss until consensus is reached and the second reviewer will screen an additional $10 \%$ of full texts (and will continue until there are no remaining conflicts).

Each article may report the results of multiple studies. We will define a "study" as a manipulative experiment that addresses a single hypothesis or research question. In the case of articles containing multiple studies, each study will be independently reviewed according to the 'Eligibility Criteria.' To account for the non-independence of studies within articles, we will include 'study' nested within 'article' as a random effect in statistical models that are a part of our meta-analyses.

In the particular case of dissertations and theses, special care will be taken to ensure that there is no duplication in our review between dissertation/thesis chapters and publications based on the same data. Peer-reviewed publications and final reports will take precedence over dissertation/thesis chapters of the same data. When dissertation/thesis chapters provide additional data that are not reported in the peer-reviewed document, these data will supplement that of the peer-reviewed document but remain a part of the same 'study'. Relevant, unpublished chapters will be treated as independent studies.

\section{Eligibility criteria}

We will use the PECO framework [28] to determine the inclusion or exclusion of each article for further review and analysis at the stages of title/abstract and full-text screening. To be included, an article/study must meet every criterion. Otherwise it will be excluded. We will 
provide a complete list of articles/studies with reason(s) for inclusion/exclusion at the full-text screening stage.

\section{Population}

All life stages of all shallow (photic zone, $\leq 80 \mathrm{~m}$ depth) scleractinian coral genera in all warm-water ocean basins $\left(20^{\circ}-30^{\circ} \mathrm{C}\right)$.

\section{Exposure}

Exposure to concentrations of suspended and/or deposited sediment of marine or terrigenous origin. For manipulative experiments conducted in either the field or laboratory, this will be the application of suspended or deposited sediment.

\section{Comparison}

Specimens experimentally exposed to suspended or deposited sediment must be compared to an appropriate experimental control in either the field or laboratory.

\section{Outcome(s)}

Specific endpoints are all physical, physiological, behavioral, developmental, and ecological responses of corals associated with exposure to deposited and/or suspended sediment. These may include but are not limited to tissue/colony mortality, bleaching, and changes in rates of growth, photosynthesis, and larval settlement/survival. Outcomes will be recorded as binary or continuous data, as reported in the study.

\section{Eligible types of study design}

Quantitative meta-analysis will be limited to the results of experimental studies that quantify the cause-effect relationship between sediment stress and coral response (including BACI-designed studies and those conducted in the field or laboratory, mesocosms, etc.), compared to the response of corals to 'ambient' or 'control' conditions. In situ, observational studies will be identified and used to contextualize the findings of manipulative experiments and the meta-analyses thereof.

\section{Study validity assessment}

We will critically appraise all studies that pass the fulltext screening process using a number of parameters including the following, which may affect a study's external validity:

- Study setting: field or laboratory;

- Temporal extent of the study: relatively long-term monitoring or short-term measurements;

- And the following, which may affect both the external and internal validity of a study; Study design: manipulative experiment or observational study; presence/extent of pseudoreplication;

- Randomization: how sediment exposure levels were assigned to coral samples; and.

- Confounding factors: degree of accounting for potential effect modifiers, if present.

Internal validity will be further assessed per the criteria outlined by Bilotta et al. [29], which adapted Cochrane's 'risk of bias' tool [30] for environmental science applications. This "Environmental-Risk of Bias Tool" assesses selection bias, performance bias, attrition bias, reporting bias, and miscellaneous bias. With this information, we will also use the "Environmental GRADE Tool" [29] to determine the overall quality (high, moderate, low, or very low) of each study. Studies with a low or very low overall grade (indicative of high susceptibility to bias) may be excluded from further analysis. We will conduct sensitivity analyses to test for the effect of these studies' inclusion/exclusion. One reviewer will assess the quality of a study. A subset of five studies will be appraised by the entire review team. Conflicting decisions of study-quality will be resolved by the entire review team. All risk assessment decisions and rationale will be recorded in the project database.

We will use these critical appraisals and tools to organize studies into groups of comparable records across which we should (and should not) meta-analyze. This process will determine the scope of inference of our meta-analysis, thus defining the extent to which our results may be applied to the diverse set of sedimentation events that occur on coral reefs.

\section{Data coding and extraction strategy}

Information from studies will be input into a data coding and extraction form [Additional file 3] and recorded in a project database that will be made available as a supporting document with the final review. The database will include study characteristics such as the sample sizes, means, and variations of coral response(s) to sediment and control conditions. When these data are not reported in the text, we will extract them from figures using opensource digitizing software that convert graph images into numerical data (e.g., Datathief [31]). When only raw data are available, we will calculate summary statistics. When information is indecipherable or missing, we will contact the corresponding author of the study for clarification. All reviewers will extract data from the same 3 studies, compare their results for any inconsistencies, and make adjustments to the protocol to improve the consistency of the data extraction process. After this pilot round, each study will have data extracted independently by one reviewer. 


\section{Potential effect modifiers/reasons for heterogeneity}

There are several factors that may cause variation in measured outcomes, information about which will be extracted and recorded in the project database. This list of effect modifiers was compiled in consultation with the Coral Tool advisory team and includes the following: study location (ocean basin, region, and site), study species and morphological form (e.g., massive, plating, branching), time/season of sediment-exposure event, sediment composition (e.g., silt-clay vs. calcareous sand) and provenance (terrestrial vs. marine), sediment dose/ concentration (and methods for measuring dose), sediment exposure duration, and possible interacting effects (e.g., light attenuation in concurrence with suspended sediment, or nutrient-enriched deposited sediment). These sources of variation are included in primary review questions $1 \mathrm{c}$ and $1 \mathrm{~d}$ and will be addressed as described in the following section (Data synthesis and presentation). While some of these effect modifiers will be categorical, others will be numerical and may require the conversion of reported units to a common standard, e.g., for deposited sediment the standard will likely be $\mathrm{mg} / \mathrm{cm}^{2} /$ day and for suspended sediment the standards will likely be either $\mathrm{mg} / \mathrm{L}$ or NTU (which cannot be converted to one another).

\section{Data synthesis and presentation}

We will present a narrative synthesis of the results of all eligible studies included in our review. This narrative will address the spatial and temporal processes that mediate coral responses to (1) deposited sediment, (2) suspended sediment, and (3) both deposited and suspended sediment. The results of observational experiments and case studies will likely be presented in this narrative format to contextualize the results of the quantitative meta-analyses applied to manipulative experiments, as described below.

We will construct tables that list the responses of corals and organize them according to sediment exposure (concentration, duration, frequency, and type), geographic location, coral taxonomy, and coral life-history stage. These tables will synthesize information from relevant studies, which will help identify types of quantitative analyses that will best address our primary review question.

For coral responses reported as binary $(0=$ no response, $1=$ response; e.g., mortality) in control and treatment conditions, the effect size for each study will be the log odds. We will perform mixed-effects logistic regressions, also known as "binomial-normal models" [32-34], with random effects for 'study' nested within 'article' and/or 'research group', if a small number of research groups contribute multiple articles. This approach will estimate the probability of an adverse response given a particular level of sediment exposure and is useful because it can provide explicit false positive, false negative, specificity, sensitivity, and ROC outputs that may inform our choice of the most robust models for regulatory purposes.

For coral responses reported as continuous variables (e.g., photosynthetic or growth rates), the effect size for each study will be the standardized difference in means. This will be calculated using Hedges' $d$ and the variance thereof [35], which is unaffected by unequal sampling variances in the paired groups (e.g., treatment and control conditions) and includes a correction factor for small sample sizes. We will then explore the relationship between effect size and stressor size with hierarchical mixed-effects models that fit both linear and non-linear exposure-response curves. This model structure will allow us to examine the overall effects on corals while accounting for within- and between-study (co)variance structures (e.g., due to random effects and other effect modifiers such as taxonomic group, geographical location, and study type). We will model deposited and suspended sediment separately and, when study-designs allow, together. To detect any publication bias in our meta-analyses, we will produce funnel plots of the effect size plotted against the standard error of the effect size [36, 37]. For studies with high susceptibility to bias (as described in Study validity assessment, above), we will conduct sensitivity analyses to test for the effect of their inclusion/exclusion from meta-analyses.

After the meta-analysis is complete, we will identify areas that will benefit most from additional empirical research. We expect two key knowledge gaps: (1) gaps in our understanding of the interactions between and among deposited sediment, suspended sediment, and other co-stressors, and (2) gaps in our understanding of regional variation in responses, especially within the U.S. Pacific Islands Region.

\section{Supplementary information}

Supplementary information accompanies this paper at https://doi. org/10.1186/s13750-020-00200-0.

Additional file 1. Filled-in ROSES checklist and meta-data form for this manuscript.

Additional file 2. Search scoping exercise and list of definitive reviews and benchmark studies.

Additional file 3. Data coding and extraction form to be used for the systematic review and meta-analysis.

\section{Acknowledgements}

The authors would like to acknowledge Dr. Malia Chow, lan Lundgren, and Gerry Davis of the NOAA PIRO for their support, feedback, and organization of team meetings during the early stages of this project. 


\section{Authors' contributions}

LJT, MJD and CJ wrote the manuscript. All authors collaborated to decide on the study questions and to design the study protocol. All authors read and approved the final manuscript.

\section{Funding}

This work was funded by the U.S. NOAA Grant NA19NMF4540068 to MJD. The project was designed by MJD and LJT in collaboration with the Coral Tool advisory team (and co-authors) CJ, SK, and DM.

\section{Availability of data and materials}

Not applicable.

\section{Ethics approval and consent to participate}

Not applicable.

\section{Consent for publication}

Not applicable.

\section{Competing interests}

The authors declare that they have no competing interests.

\section{Author details}

${ }^{1}$ Hawai'i Institute of Marine Biology, Kăne'ohe, Hawai'i 96744, USA. ${ }^{2}$ Systematic Ecology, Bethesda, MD 20817, USA. ${ }^{3}$ NOAA Pacific Islands Regional Office, Honolulu, Hawai'i 96860, USA. ${ }^{4}$ Dwayne Minton Consulting, Monmouth, Oregon 97361, USA.

Received: 19 February 2020 Accepted: 29 July 2020 Published online: 08 August 2020

\section{References}

1. Jones P, Murray R, Vestergaard O. Marine Protected Areas: Securing Benefits for Sustainable Development; Frontiers 2017: Emerging Issues of Environmental Concern. 2017.

2. Bellwood DR, Hughes TP, Folke CS, Nyström M. Confronting the coral reef crisis. Nature. 2004:429:827-33.

3. Côté IM, Gill JA, Gardner TA, Watkinson AR. Measuring coral reef decline through meta-analyses. Philos Trans R Soc B Biol Sci. 2005;360:385-95

4. Hughes TP, Kerry JT, Baird AH, Connolly SR, Dietzel A, Eakin CM, et al. Global warming transforms coral reef assemblages. Nature. 2018:556:492-6.

5. Carpenter KE, Abrar M, Aeby G, Aronson RB, Banks S, Bruckner A, et al. One-third of reef-building corals face elevated extinction risk from climate change and local impacts. Science. 2008;321:560-3.

6. Mumby PJ, Broad K, Brumbaugh DR, Dahlgren CP, Harborne AR, Hastings $A$, et al. Coral reef habitats as surrogates of species, ecological functions, and ecosystem services. Conserv Biol. 2008;22:941-51.

7. Spalding M, Burke L, Wood SA, Ashpole J, Hutchison J, Ermgassen P. Mapping the global value and distribution of coral reef tourism. Mar Policy. 2017:82:104-13.

8. CFR 230.44: Clean Water Act Section 404(b)(1) Guidelines for Specification of Disposal Sites for Dredged or Fill Material. USA; 2010 p. 253-306.

9. FR 53852: Endangered and Threatened Wildlife and Plants: Final Listing Determinations on Proposal To List 66 Reef-Building Coral Species and To Reclassify Elkhorn and Staghorn Corals. USA; 2014 p. 53852-4123.

10. Sheppard CRC, Davy SK, Pilling GM, Graham NAJ. The biology of coral reefs. 2nd ed. Oxford: Oxford University Press; 2018.

11. CFR 660.75: Essential Fish Habitat (EFH). USA; 2012 p. 129-33.

12. Mumby PJ, Steneck RS. Coral reef management and conservation in light of rapidly evolving ecological paradigms. Trends Ecol Evol. 2008:23:555-63.

13. Carilli JE, Norris RD, Black BA, Walsh SM, McField M. Local stressors reduce coral resilience to bleaching. PLoS ONE. 2009:4:1-5.

14. Gurney GG, Melbourne-Thomas J, Geronimo RC, Aliño PM, Johnson CR. Modelling coral reef futures to inform management: Can reducing local-scale stressors conserve reefs under climate change? PLOS ONE. 2013:8:1-17.
15. Erftemeijer PLA, Riegl B, Hoeksema BW, Todd PA. Environmental impacts of dredging and other sediment disturbances on corals: a review. Mar Pollut Bull. 2012;64:1737-65.

16. Fabricius KE. Effects of terrestrial runoff on the ecology of corals and coral reefs: review and synthesis. Mar Pollut Bull. 2005:50:125-46.

17. Jones R, Bessell-Browne P, Fisher R, Klonowski W, Slivkoff M. Assessing the impacts of sediments from dredging on corals. Mar Pollut Bull. 2016;102:9-29.

18. Rogers CS. Responses of coral reefs and reef organisms to sedimentation. Mar Ecol Prog Ser. 1990;62:185-202.

19. Risk MJ. Assessing the effects of sediments and nutrients on coral reefs. Curr Opin Environ Sustain. 2014;7:108-17.

20. Jones R, Ricardo GF, Negri AP. Effects of sediments on the reproductive cycle of corals. Mar Pollut Bull. 2015;100:13-33.

21. Collaboration for Environmental Evidence. Guidelines and Standards fo Evidence Synthesis in Environmental Management. version 5. Pullin AS Frampton GK, Livoreil B, Petrokofsky G, editors. 2018.

22. Pullin AS, Stewart GB. Guidelines for systematic review in conservation and environmental management. Conserv Biol. 2006;20:1647-56.

23. Haddaway NR, Macura B, Whaley P, Pullin AS. ROSES for Systematic Review Protocols. https://www.roses-reporting.com. Accessed 3 Aug 2020

24. Harzing, AW. Publish or Perish. http://www.harzing.com/pop.htm. Accessed 6 June 2020.

25. Wallace BC, Small K, Brodley CE, Lau J, Trikalinos TA. Deploying an interactive machine learning system in an evidence-based practice center: abstrackr. Proc. of the ACM International Health Informatics Symposium $(\mathrm{IHI}), 2012$.

26. Gates A, Johnson C, Hartling L. Technology-assisted title and abstract screening for systematic reviews: A retrospective evaluation of the Abstrackr machine learning tool. Syst Rev. 2018:7:1-9.

27. Rathbone J, Hoffmann T, Glasziou P. Faster title and abstract screening? Evaluating Abstrackr, a semi-automated online screening program for systematic reviewers. Syst Rev. 2015;4:1-7

28. Morgan RL, Whaley P, Thayer KA, Schünemann HJ. Identifying the PECO: a framework for formulating good questions to explore the association of environmental and other exposures with health outcomes. Environ Int. 2018:121:1027-31.

29. Bilotta GS, Milner AM, Boyd IL. Quality assessment tools for evidence from environmental science. Environ Evid. 2014;3:1-14.

30. Higgins JPT, Altman DG, Gøtzsche PC, Jüni P, Moher D, Oxman AD, et al. The Cochrane Collaboration's tool for assessing risk of bias in randomised trials. BMJ. 2011;343:1-9.

31. DataThief III. https://datathief.org. Accessed 13 Feb 2020

32. Stijnen T, Hamza TH, Ozdemir P. Random effects meta-analysis of event outcome in the framework of the generalized linear mixed model with applications in sparse data. Tut in Biostat. 2010;29:3046-67.

33. Simmonds MC, Higgins JPT. A general framework for the use of logistic regression models in meta-analysis. Stat Meth in Med Res. 2014;25:2858-77

34. Bakbergenuly I, Kulinskaya E. Meta-analysis of binary outcomes via generalized linear mixed models: a simulation study. BMC Med Res Methodol. 2018;18:70.

35. Hedges LV, Olkin I. Statistical Methods for Meta-Analysis. London: Academic Press, Inc.: 1985.

36. Egger M, Smith GD, Schneider M, Minder C. Bias in meta-analysis detected by a simple, graphical test. Br Med J. 1997;315:629-34.

37. Palmer AR. Detecting publication bias in meta-analyses: A case study of fluctuating asymmetry and sexual selection. Am Nat. 1999;154:220-33.

38. Western Australia Marine Science Institute's Dredging Science Node. https://www.wamsi.org.au/dredging-science-node\%0D\%0A. Accessed 13 Feb 2020.

\section{Publisher's Note}

Springer Nature remains neutral with regard to jurisdictional claims in published maps and institutional affiliations. 